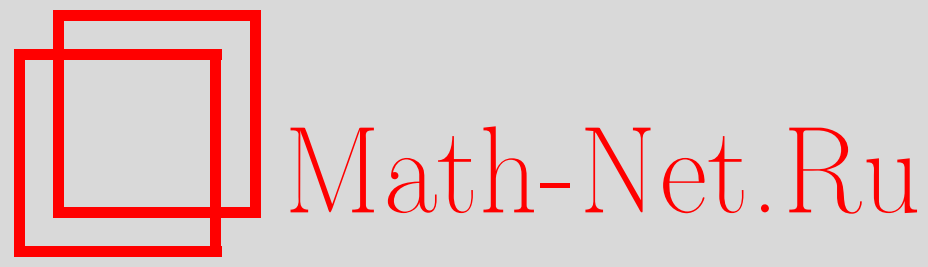

А. И. Петров, Случайные контекстно свободные грамматики: надкритический случай с ненулевой вероятностью вырождения, Теория вероятн. и ее примен., 2002, том 47, выпуск 4, 794-803

DOI: https://doi.org/10.4213/tvp3785

Использование Общероссийского математического портала Math-Net.Ru подразумевает, что вы прочитали и согласны с пользовательским соглашением

http: //www . mathnet.ru/rus/agreement

Параметры загрузки:

IP : 3.85 .7 .115

26 апреля 2023 г., 13:16:30

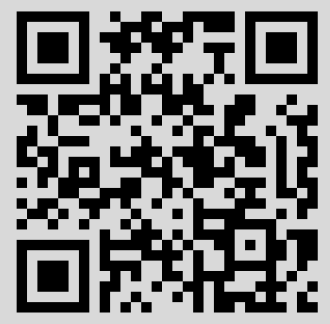


3. Лебедев A. В. Экстремумы полей дробового шума. - Фундам. и прикл. матем., 2001, т. 7, № 4, c. 1081-1090.

4. Феллер В. Введение в теорию вероятностей и ее приложения. Т. 2. М.: Мир, 1984, $752 \mathrm{c}$.

5. Westcott $M$. On the existence of a generalized shot-noise process. - Studies in Probability and Statistics. Amsterdam: North-Holland, 1976, p. 74-88.

6. Рюэль Д. Статистическая механика. М.: Мир, 1971, 368 с.

7. Heinrich L., Schmidt $V$. Normal convergence of multidimensional shot noise and rates of this convergence. - Adv. Appl. Probab., 1985, v. 17, № 3, p. 709-730.

8. Cline D. B. H. Infinite series of random variables with regularly varying tails. Technical report 83-24. Inst. Appl. Math. Univ. British Columbia, 1983, 24 p.

9. Resnick S.I. Extreme Values, Regular Variation, and Point Processes. New York: Springer-Verlag, 1987, $320 \mathrm{p}$.

10. Esary J., Prochan F., Walkup D. Association of random variables with applications. Ann. Math. Statist., 1967, v. 38, № 5, p. 1466-1474.

11. Бахтин Ю. Ю. Закон повторного логарифма для решения уравнения Бюргерса со случайными начальными данными. - Матем. заметки, 1998, т. 64, № 6, с. 812823.

Поступила в редакцию 20.I. 2000

(C) $2002 \mathrm{r}$

ПЕТРОВ А. И.*

\title{
СЛУЧАЙНЫЕ КОНТЕКСТНО СВОБОДНЫЕ ГРАММАТИКИ: НАДКРИТИЧЕСКИЙ СЛУЧАЙ С НЕНУЛЕВОЙ ВЕРОЯТНОСТЬЮ ВЫРОЖДЕНИЯ
}

\begin{abstract}
Случайные грамматики были введены в информатике, но их изучение с использованием методов статистической физики таких, как исследования термодинамического предела, техника кластерных разложений и т.д., началось недавно, см. [1]. Для контекстно-свободных грамматик в надкритическом случае с нулевой вероятностью вырождения (когда символы не умирают) в [7] исследовалось поведение при больших временах, доказывалось существование различных предельных мер и изучалась связь между ними. В данной работе мы распространим основные результаты [7] на надкритический случай с ненулевой вероятностью вырождения.

Ключевые слова и фразы: случайная контекстно свободная грамматика, ветвящийся процесс, надкритическая область, термодинамический предел.
\end{abstract}

Обозначим через $S=\left\{s_{1}, \ldots, s_{|S|}\right\}$ конечное множество, которое мы будем называть алфавитом. Словом $\alpha$ над алфавитом $S$ назовем любую линейно-упорядоченную последовательность символов из $S$. Длину слова $\alpha$ будем обозначать $|\alpha|$. Через $e$ обозначим пустое слово, $|e|=0$. Множество всех конечных слов над алфавитом $S$, включая пустое слово, мы будем обозначать через $S^{*} ; S^{+}=S^{*} \backslash\{e\}$.

Для конечных слов $\alpha=x_{1} \ldots x_{n}, \beta=y_{1} \ldots y_{m}, m, n>0, x_{i}, y_{j} \in S, i=1, \ldots, n$, $j=1, \ldots, m$, их композицией $\alpha \beta$ называется слово

$$
\alpha \beta=x_{1} \ldots x_{n} y_{1} \ldots y_{m}, \quad \alpha e=e \alpha=\alpha .
$$

* Московский государственный университет им. М.В. Ломоносова, Лаборатория больших случайных систем, Ленинские горы, 119992 Москва, Россия; e-mail: aipetrov@lbss.math.msu.su 
Слово $\alpha \in S^{*}$ является подсловом слова $\gamma \in S^{*}$, если $\gamma=\beta \alpha \delta$, где $\beta, \delta \in S^{*}$.

Рассмотрим марковский процесс $Z_{t}^{[\gamma]}$ с дискретным временем $t \geqslant 0$, имеющий в качестве множества состояний множество всех слов над алфавитом $S$. Начальное состояние при $t=0$ есть фиксированное бесконечное слово $\gamma \equiv \gamma(0)=x_{1}^{[\gamma]}(0) x_{2}^{[\gamma]}(0) \ldots$, где $x_{i}^{[\gamma]}(0) \in S, i=1,2, \ldots$. Состоянием $Z_{t}^{[\gamma]}$ в момент времени $t \geqslant 0$ является слово

$$
\gamma(t)=x_{1}^{[\gamma]}(t) x_{2}^{[\gamma]}(t) \ldots, \quad \text { где } \quad x_{i}^{[\gamma]}(t) \in S, i=1,2, \ldots .
$$

Переходные вероятности определяются следующим образом: каждый символ $x$ слова $\gamma(t)$ в момент времени $t \geqslant 0$, независимо от других символов, заменяется на слово $\alpha \in S^{*}$ с вероятностью $\mathbf{P}\{x \rightarrow \alpha\}$. Мы будем предполагать выполнение следующих условий:

$$
\begin{gathered}
\forall x, s \in S: \mathbf{P}\{x \rightarrow s\}>0, \\
\exists K \geqslant 1: \forall x \in S: \sum_{\alpha \in S^{*},|\alpha| \leqslant K} \mathbf{P}\{x \rightarrow \alpha\}=1 .
\end{gathered}
$$

Очевидно, что с вероятностью единица в любой момент времени $t \geqslant 0$ длина слова $\gamma(t)$ бесконечна. Случай $\forall x \in S: \mathbf{P}\{x \rightarrow \varnothing\}=0$ был рассмотрен в [7]. Нас будет интересовать структура слова $\gamma(t)$ при $t \rightarrow \infty$.

Наряду с процессом $Z_{t}^{[\gamma]}$ рассмотрим процессы $Z_{t}^{[k]}, k=1, \ldots,|S|$, с теми же перехоцными вероятностями, что и у $Z_{t}^{[\gamma]}$, но имеющие в качестве начального состояния один символ $\gamma^{[k]}(0) \equiv x_{1}^{[k]}(0)=s_{k} \in S$. Состояние $Z_{t}^{[k]}$ в момент $t \geqslant 0$ есть слово длины $n^{[k]}(t)$ :

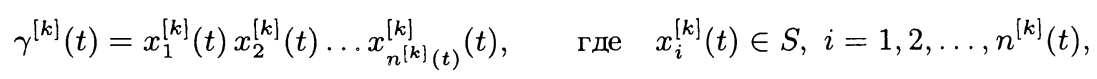

$n^{[k]}(t)=0$ при $\gamma^{[k]}(t)=\varnothing$. Введем векторы размерности $|S|: \mathbf{1}=(1, \ldots, 1), \mathbf{0}=$ $(0, \ldots, 0)$ и $\mathbf{e}_{j}=(0, \ldots, 0,1,0, \ldots, 0)$ - где 1 стоит на $j$-м месте.

Обозначим через $n(\alpha, \gamma), \alpha \in S^{+}, \gamma \in S^{*}$, число подслов $\alpha$ в слове $\gamma$. Введя

$$
N_{t}^{[k]}\left(s_{i}\right) \equiv n\left(s_{i}, \gamma^{[k]}(t)\right), \quad s_{i} \in S, \quad i, k=1, \ldots,|S|,
$$

получим, что $\vec{N}_{t}^{[k]} \equiv\left(N_{t}^{[k]}\left(s_{1}\right), \ldots, N_{t}^{[k]}\left(s_{|S|}\right)\right)$ есть ветвящийся процесс с $|S|$ типами частиц (см. [2], [3], [5]), начальным состоянием $\mathbf{e}_{k}$ и

$$
p^{[k]}\left(j_{1}, \ldots, j_{|S|}\right)=\sum_{\alpha \in S^{*}: n\left(s_{1}, \alpha\right)=j_{1}, \ldots, n\left(s_{|S|}, \alpha\right)=j_{|S|}} \mathbf{P}\left\{s_{k} \rightarrow \alpha\right\},
$$

где $p^{[k]}\left(j_{1}, \ldots, j_{|S|}\right), k=1, \ldots,|S|$, есть вероятность того, что из символа $s_{k} \in S$ появилось за один момент времени $j_{1} \geqslant 0$ символов $s_{1}, \ldots, j_{|S|} \geqslant 0$ символов $s_{|S|}$. Пусть $\mathscr{C}_{|S|}=[0,1] \times \cdots \times[0,1]-$ единичный куб в $\mathbf{R}^{|S|}-|S|$-мерном пространстве действительных чисел и $\mathbf{x}=\left(x_{1}, \ldots, x_{|S|}\right) \subset \mathscr{C}_{|S|}$, тогда $\vec{N}_{1}^{[k]}$ имеет производящую функцию

$$
f^{[k]}(\mathbf{x}) \equiv f^{[k]}\left(x_{1}, \ldots, x_{|S|}\right)=\sum_{j_{1}, \ldots, j_{|S|}=0}^{\infty} p^{[k]}\left(j_{1}, \ldots, j_{|S|}\right) x_{1}^{j_{1}} \cdots x_{|S|}^{j_{|S|}} .
$$

Производящую функцию процесса $\vec{N}_{t}^{[k]}$ при $t>0$ обозначим $f_{t}^{[k]}(\mathbf{x})$ и введем $\mathbf{f}_{t}(\mathbf{x})=$ $\left(f_{t}^{[1]}(\mathbf{x}), \ldots, f_{t}^{[|S|]}(\mathbf{x})\right), \mathbf{f}_{0}(\mathbf{x}) \equiv \mathbf{x}, \mathbf{f}(\mathbf{x}) \equiv \mathbf{f}_{1}(\mathbf{x})=\left(f^{[1]}(\mathbf{x}), \ldots, f^{[|S|]}(\mathbf{x})\right)$. Известно (см. [2], [3], [5]), что при $t \geqslant 0: \mathbf{f}_{t+1}(\mathbf{x})=\mathbf{f}\left(\mathbf{f}_{t}(\mathbf{x})\right)$. В том случае, когда начальное состояние процесса $\vec{N}_{t}^{[k]}$ для нас неважно, мы будем опускать верхний индекс и писать $\vec{N}_{t}$. Обозначим через $M=\left(m_{i j}\right), m_{i j}=\mathbf{E} N_{1}^{[i]}\left(s_{j}\right)$, матрицу средних ветвящегося процесса $\vec{N}_{t}$. Известно (см. [2], [3], [5]), что $\mathbf{E}\left(\vec{N}_{t+T} \mid \vec{N}_{T}\right)=\vec{N}_{T} M^{t}$, где $t, T \geqslant 0$. Мы будем предполагать, что выполнены следующие условия. 
П р е д п о л о ж е н и е $1 . \vec{N}_{t}$ есть надкритический ветвяшийся процесс (т.е. максимальное по модулю собственное значение $\varrho$ его матрищы средних $M$ больше 1) (см. [2], [3], [5]) и вероятности подстановок удовлетворяют (1)-(2).

Назовем матрицу $M=\left(m_{i j}\right)$ строго положительной $(M>0)$, если $\min _{i, j}\left(m_{i j} t\right)>0$.

О п р е д е л е н и е 1 . Ветвящийся процесс $\vec{N}_{t}$ называется положительно регулярныц, если для его матрицы средних $M$ выполнено: $\exists n>0, M^{n}>0$.

Ветвящийся процесс $\vec{N}_{t}$ называется сингулярным, если его производящая функция $\mathbf{f}(\mathbf{x})$ имеет вид $\mathbf{f}(\mathbf{x})=\mathbf{x} C$, где $C$ - некоторая постоянная матрица с неотрицательными элементами. В этом случае каждый объект имеет ровно одного потомка.

Заметим, что из предположения 1 следует, что $\vec{N}_{t}$ есть положительно регулярный и несингулярный ветвящийся процесс.

О п р е д е л е н и е 2. Для любого слова $\alpha=y_{1} \ldots y_{m} \in S^{+}, y_{1}, \ldots, y_{m} \in S$, $m>0$, и $t>0$ обозначим через $p^{[\gamma]}(\alpha ; t)-|\alpha|$-мерное распределение процесса $Z_{t}^{[\gamma]}$ :

$$
p^{[\gamma]}(\alpha ; t)=\mathbf{P}\left\{x_{1}^{[\gamma]}(t)=y_{1}, \ldots, x_{m}^{[\gamma]}(t)=y_{m}\right\} .
$$

О п р е д е л е н и е 3 . Для любого слова $\alpha \in S^{+}$и $t>0$ введем

$$
\mu^{[k]}(\alpha ; t)=\frac{\mathbf{E} n\left(\alpha, \gamma^{[k]}(t)\right)}{\mathbf{E} n^{[k]}(t)}, \quad k=1, \ldots,|S| .
$$

\section{Основные результаты.}

Теорема 1. Если выполнено предположение 1, то для любого слова $\alpha \in S^{+} u$ любого начального слова $\gamma,|\gamma|=\infty$, существует предел

$$
\pi^{[\gamma]}(\alpha)=\lim _{t \rightarrow \infty} p^{[\gamma]}(\alpha ; t) .
$$

Теорема 2. Если $\vec{N}_{t}$ есть надкритический ветвящийся прочесс со строго положительной матричей средних и вероятности подстановок удовлетворяют (2), то для любого слова $\alpha \in S^{+}$существует предел

$$
\mu(\alpha)=\lim _{t \rightarrow \infty} \mu^{[k]}(\alpha ; t), \quad k=1, \ldots,|S|,
$$

не зависяший от $k$, причем $\forall \alpha \in S^{+}: \mu(\alpha)>0$.

Теорема 3. Если выполнено предположение 1, то для любого слова $\alpha \in S^{+} u$ любого $\varepsilon>0$ сушествуют $L(\varepsilon, \alpha), t(\varepsilon, \alpha)>0$ такие, что для любых $L>L(\varepsilon, \alpha)$, $t>t(\varepsilon, \alpha), j>0$ и любого начального слова $\gamma,|\gamma|=\infty$, выполнено:

$$
\mathbf{P}\left\{\left|\frac{n\left(\alpha, x_{j}^{[\gamma]}(t) \cdots x_{j+L-1}^{[\gamma]}(t)\right)}{L-|\alpha|+1}-\mu(\alpha)\right|>\varepsilon\right\}<\varepsilon
$$

Теорема 4. Если выполнено предположение 1, то для любого слова $\alpha=$ $y_{1} \ldots y_{m} \in S^{+}, y_{1}, \ldots, y_{m} \in S, m>0$, существует предел

$$
\lim _{j \rightarrow \infty} \lim _{t \rightarrow \infty} \mathbf{P}\left\{x_{j}^{[\gamma]}(t)=y_{1}, \ldots, x_{j+m-1}^{[\gamma]}(t)=y_{m}\right\}=\mu(\alpha),
$$

не зависящий от начального слова $\gamma,|\gamma|=\infty$.

Следствие. Если выполнено предположение 1, то $\mu(\alpha)$ является вероятностной мерой на словах фиксированной длины, т.е. $\mu(\alpha)$ неотричательна, аддитивна и для любого $m>0: \sum_{\alpha \in S^{+}:|\alpha|=m} \mu(\alpha)=1$.

Но без предположения 1 , как было показано в [7], теорема 4 неверна.

Ниже мы приводим доказательства теорем 1 и 2 , эти доказательства не используют результатов из [7] и, более того, потребовали применения новых методов. Доказательства теорем 3 и 4 аналогичны доказательствам соответствующих теорем для случая $\forall s \in S: \mathbf{P}\{s \rightarrow \varnothing\}=0$ из работы [7] и мы опускаем их. 
Доказательство теоремы 1. Введем обозначения: для $i=1, \ldots,|S|$ и $t>0$

$$
\begin{aligned}
q^{[i]} & =\mathbf{P}\left\{\exists t>0: \vec{N}_{t}^{[i]}=\mathbf{0}\right\}, \quad q^{[i]}(t)=\mathbf{P}\left\{\vec{N}_{t}^{[i]}=\mathbf{0}\right\}, \\
\mathbf{q} & =\left(q^{[1]}, \ldots, q^{[|S|]}\right), \quad \mathbf{q}(t)=\left(q^{[1]}(t), \ldots, q^{[|S|]}(t)\right), \\
D & =\left(d_{i j}\right)_{i, j=1, \ldots,|S|}, \quad \text { где } \quad d_{i j}=\left.\frac{d f^{[i]}(\mathbf{x})}{d x_{j}}\right|_{\mathbf{x}=\mathbf{q}} .
\end{aligned}
$$

Для векторов $\mathbf{x}=\left(x_{1}, \ldots, x_{|S|}\right)$ и $\mathbf{y}=\left(y_{1}, \ldots, y_{|S|}\right), \mathbf{x}, \mathbf{y} \in \mathbf{R}^{|S|}$, введем следующие обозначения: $|\mathbf{x}|=\max _{i=1, \ldots,|S|}\left|x_{i}\right| ; \mathbf{x}<\mathbf{y}(\mathbf{x} \leqslant \mathbf{y})$, если $x_{i}<y_{i}\left(x_{i} \leqslant y_{i}\right)$ для $i=1, \ldots,|S|$; для $\mathbf{x}>0, \mathbf{y} \geqslant \mathbf{0}: \mathbf{x}^{\mathbf{y}}=x_{1}^{y_{1}} \cdots x_{|S|}^{y_{|S|}}$.

В работе $[4$, с. 568$]$ была доказана следующая теорема, которая нам понадобится в дальнейшем.

Теорема. Если $\vec{N}_{t}$ есть надкритический ветвяиийся прочесс и существует $\tau$, $0<\tau<1$, такое, что $D^{n} \tau^{-n}$ сходится $\kappa$ ненулевой, конечной матриче при $n \rightarrow \infty$, то существует вектор-функиия $\mathbf{Q}: \mathscr{C}_{|S|} \rightarrow \mathbf{R}^{|S|}$ такая, что для $0 \leqslant \mathbf{x}<\mathbf{1}$

$$
\begin{array}{cl}
\frac{\mathbf{f}_{n}(\mathbf{x})-\mathbf{q}}{\tau^{n}} \rightarrow \mathbf{Q}(\mathbf{x}) \quad n p u & n \rightarrow \infty, \quad \mathbf{Q}(\mathbf{q})=\mathbf{0}, \\
\mathbf{0} \leqslant \mathbf{Q}(\mathbf{x})<\infty \quad \text { длs } \mathbf{q}<\mathbf{x}<\mathbf{1}, \quad & |\mathbf{Q}(\mathbf{x})|<\infty \quad \partial \Omega я \mathbf{0} \leqslant \mathbf{x}<\mathbf{1} .
\end{array}
$$

Лемма 1. Если выполнено предположение 1, то выполнены все условия предыдущей теоремы и, следовательно, верно (6).

Д о к а з а т е л ь с т в о. Из (1), (2), (4) и (5) следует, что матрица $D$ строго положительная, и в силу теоремы Перрона-Фробениуса (см. [3, с. 185]) достаточно проверить, что ее максимальное по модулю собственное значение строго меньше единицы. в виде

Учитывая (2) и используя разложение Тейлора для $\mathbf{f}(\mathbf{x}), \mathbf{x} \in \mathscr{C}_{|S|}$, представим $\mathbf{f}(\mathbf{x})$

$$
\mathbf{f}(\mathbf{x})=\mathbf{f}(\mathbf{q})+(\mathbf{x}-\mathbf{q}) B+\mathbf{g}(\mathbf{x})
$$

где $B$ есть транспонированная матрица $D$ (а значит, $B>0$ ) и компоненты $\mathrm{g}^{[l]}(\mathbf{x})$, $l=1, \ldots,|S|$, функции $\mathbf{g}(\mathbf{x})$ суть линейные комбинации с неотрицательными коэффициентами членов вида

$$
\left.\left(x_{1}-q^{[1]}\right)^{i_{1}} \cdots\left(x_{|S|}-q^{[|S|]}\right)^{i_{|S|}} \frac{d f^{[l]}(\mathbf{x})}{d x_{1}^{i_{1}} \cdots d x_{|S|}^{i_{|S|}}}\right|_{\mathbf{x}=\mathbf{q}},
$$

где $i_{j} \geqslant 0, j=1, \ldots,|S|$, и $1<i_{1}+\cdots+i_{|S|} \leqslant K$, но из (1), (2), (4) и (5) следует, что частные производные любого порядка от $\mathbf{f}(\mathbf{x})$ по $x_{1}, \ldots, x_{|S|}$ неотрицательны в точках $\mathbf{x}, \mathbf{0}<\mathbf{x}<\mathbf{1}$ и, значит,

$$
\forall \mathbf{x}, \mathbf{q}<\mathbf{x}<1: \mathbf{g}(\mathbf{x}) \geqslant 0 .
$$

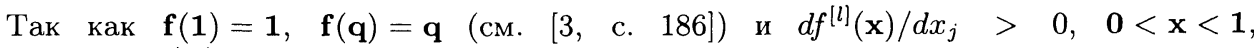
$j, l=1, \ldots,|S|$, то

$$
\forall \mathbf{x}, \mathbf{q}<\mathbf{x}<\mathbf{1}: \mathbf{q}<\mathbf{f}(\mathbf{x})<\mathbf{1}
$$

Тогда из $(8),(9)$ и $\mathbf{f}_{t}(\mathbf{x})=\mathbf{f}\left(\mathbf{f}_{t-1}(\mathbf{x})\right)($ см. $[5$, с. 36]) следует, что

$$
\forall k \geqslant 0, \forall \mathbf{x}, \mathbf{q}<\mathbf{x}<\mathbf{1}: \mathbf{g}\left(\mathbf{f}_{k}(\mathbf{x})\right) \geqslant \mathbf{0} .
$$

Это неравенство нам понадобится ниже. Проитерируем $(7)$ с учетом $\mathbf{f}_{t}(\mathbf{x})=\mathbf{f}\left(\mathbf{f}_{t-1}(\mathbf{x})\right)$ и $\mathbf{f}(\mathbf{q})=\mathbf{q}$ :

$$
\forall t>0: \mathbf{f}_{t}(\mathbf{x})=\mathbf{q}+(\mathbf{x}-\mathbf{q}) B^{t}+\sum_{j=0}^{t-1} \mathbf{g}\left(\mathbf{f}_{t-1-j}(\mathbf{x})\right) B^{j}
$$


Взяв $\mathbf{x}: \mathbf{q}<\mathbf{x}<\mathbf{1}$ и переходя к пределу при $t \rightarrow \infty$, с учетом соотношений $\forall \mathbf{x}$, $\mathbf{0} \leqslant \mathbf{x}<\mathbf{1 :} \lim _{t \rightarrow \infty} \mathbf{f}_{t}(\mathbf{x})=\mathbf{q}($ см. $[3$, с. 186]) и $\mathbf{f}(\mathbf{q})=\mathbf{q}$ получим

$$
\lim _{t \rightarrow \infty}\left((\mathbf{x}-\mathbf{q}) B^{t}+\sum_{j=0}^{t-1} \mathbf{g}\left(\mathbf{f}_{t-1-j}(\mathbf{x})\right) B^{j}\right)=\mathbf{0} .
$$

Как упоминалось выше, $B>0$, значит, учитывая (10) и то, что $\mathbf{x}-\mathbf{q}>\mathbf{0}$, из (11) получим $\lim _{t \rightarrow \infty} B^{t}=0$. Применяя теорему Перрона-Фробениуса к строго положительной матрице $B$, мы видим, что это возможно только в том случае, когда максимальное по модулю собственное значение матрицы $B$ меньше единицы. Что и требовалось доказать.

Лемма 2. Если выполнено предположение 1, то для любого $j>0$ существуют $C \equiv C(j)>0$ и $\tau<1$ такие, что для любого $t>0 u \mathbf{k}=\left(k_{1}, \ldots, k_{|S|}\right)$

$$
\sum_{\mathbf{k}: 0<k_{1}+\cdots+k_{|S|} \leqslant j} \mathbf{P}\left\{\vec{N}_{t}^{[l]}=\mathbf{k}\right\}<C \tau^{t}, \quad l=1, \ldots,|S| .
$$

Д о к а з а т ел ь с т в о. Выберем $\mathbf{x}=\left(x_{1}, \ldots, x_{|S|}\right)$ :

$$
0<x_{i}<1, \quad i=1, \ldots,|S| .
$$

Тогда из леммы 1 следует, что сушествует $c \equiv c(\mathbf{x})>0$ такое, что для любого $t>0$

$$
\frac{f_{t}^{[l]}(\mathbf{x})-q^{[l]}}{\tau^{t}}<c, \quad l=1, \ldots,|S| .
$$

Домножая на $\tau^{t}$ и расписывая $f_{t}^{[l]}(\mathbf{x})$, приходим к оценке

$$
\sum_{\mathbf{k}: k_{1}+\cdots+k_{|S|}>0} \mathbf{P}\left\{\vec{N}_{t}^{[l]}=\mathbf{k}\right\} \mathbf{x}^{\mathbf{k}}+q^{[l]}(t)-q^{[l]}<c \tau^{t} .
$$

Учитывая соотношения $\mathbf{q}(t)-\mathbf{q} \leqslant \mathbf{0}$ и $q^{[l]}(t)=\mathbf{P}\left\{\vec{N}_{t}^{[l]}=0\right\}=f_{t}^{[l]}(0), l=1, \ldots,|S|$ (см. $[3$, с. 183,186$])$, из леммы 1 получаем, что существует $d>0$ такое, что для любого $t>0$

$$
-d \tau^{t}<q^{[l]}(t)-q^{[l]} \leqslant 0, \quad l=1, \ldots,|S| .
$$

Подставляя это в (13), получим

$$
\left(\sum_{\mathbf{k}: 0<k_{1}+\cdots+k_{|S|} \leqslant j}+\sum_{\mathbf{k}: k_{1}+\cdots+k_{|S|}>j}\right) \mathbf{P}\left\{\vec{N}_{t}^{[l]}=\mathbf{k}\right\} \mathbf{x}^{\mathbf{k}}<(c+d) \tau^{t} .
$$

Возьмем $a \equiv a(\mathbf{x}, j)=\min _{\mathbf{k}: 0<k_{1}+\cdots+k_{|S|} \leqslant j}\left\{\mathbf{x}^{\mathbf{k}}\right\}$. Из (12) следует, что $a>0$, а значит, из (14) получаем, что

$$
0 \leqslant \sum_{\mathbf{k}: 0<k_{1}+\cdots+k_{|S|} \leqslant j} \mathbf{P}\left\{\vec{N}_{t}^{[l]}=\mathbf{k}\right\}<\frac{(c+d)}{a} \tau^{t} .
$$

Лемма 2 доказана.

Для любого слова $\alpha=z_{1} \ldots z_{|\alpha|}, z_{1}, \ldots, z_{|\alpha|} \in S,|\alpha| \geqslant 1$, введем

$$
\begin{aligned}
r^{[i]}(\alpha, 0) & =0 \\
\forall t>0: r^{[i]}(\alpha, t) & =\mathbf{P}\left\{n^{[i]}(t) \geqslant|\alpha| ; x_{1}^{[i]}(t)=z_{1}, \ldots, x_{|\alpha|}^{[i]}(t)=z_{|\alpha|}\right\},
\end{aligned}
$$

где $i=1, \ldots,|S|$. Введем также вектор $\vec{r}(\alpha, t)$ с компонентами $r^{[i]}(\alpha, t), i=1, \ldots,|S|$. Докажем существование предела $\vec{r}(\alpha, t)$ при $t \rightarrow \infty$. Рассмотрим сначала случай $\alpha=s, s \in S$, т.е. $|\alpha|=1$. 
Для $r^{[i]}(\alpha, t), t>1, i=1, \ldots,|S|$, напишем рекуррентные уравнения, аналогичные обратным уравнениям Колмогорова для ветвяшихся процессов:

$$
\begin{aligned}
r^{[i]}(\alpha, t)=\sum_{j=1}^{|S|}\left(\sum_{\beta \in S^{*}}(\right. & \mathbf{P}\left\{s_{i} \rightarrow s_{j} \beta\right\}+\sum_{m=1}^{K-1} \sum_{i_{1}, \ldots, i_{m}=1}^{|S|} \mathbf{P}\left\{s_{i} \rightarrow s_{i_{1}} \ldots s_{i_{m}} s_{j} \beta\right\} \\
& \left.\left.\times q^{\left[i_{1}\right]}(t-1) \cdots q^{\left[i_{m}\right]}(t-1)\right)\right) r^{[j]}(\alpha, t-1) .
\end{aligned}
$$

Введем матрицы $Q$ и $E(t)$ размера $|S| \times|S|$ с элементами

$$
\begin{aligned}
q_{i j} & =\sum_{\beta \in S^{*}}\left(\mathbf{P}\left\{s_{i} \rightarrow s_{j} \beta\right\}+\sum_{m=1}^{K-1} \sum_{i_{1}, \ldots, i_{m}=1}^{|S|} \mathbf{P}\left\{s_{i} \rightarrow s_{i_{1}} \ldots s_{i_{m}} s_{j} \beta\right\} q^{\left[i_{1}\right]} \cdots q^{\left[i_{m}\right]}\right) \\
e_{i j}(k) & =\sum_{\beta \in S^{*}} \sum_{m=1}^{K-1} \sum_{i_{1}, \ldots, i_{m}=1}^{|S|} \mathbf{P}\left\{s_{i} \rightarrow s_{i_{1}} \ldots s_{i_{m}} s_{j} \beta\right\}\left(q^{\left[i_{1}\right]} \cdots q^{\left[i_{m}\right]}-q^{\left[i_{1}\right]}(k) \cdots q^{\left[i_{m}\right]}(k)\right)
\end{aligned}
$$

соответственно, $i, j=1, \ldots,|S|, k>0$. Заметим, что из (1) следует, что $Q-$ строго положительная матрица.

Теперь рекуррентные уравнения для $r^{[i]}(\alpha, t)$ можно переписать в матричном виде:

$$
\vec{r}(s, t)=(Q-E(t-1)) \vec{r}(s, t-1),
$$

или, применяя индукцию,

$$
\vec{r}(s, t)=\prod_{k=2}^{t}(Q-E(k-1)) \vec{r}(s, 1) .
$$

Так как для $k>0: q^{[i]}(k) \leqslant q^{[i]}$ и $q^{[i]}(k)=f_{k}^{[i]}(0), 1 \leqslant i \leqslant|S|$, то из (15) и леммы 1 следует оценка

$$
\forall k>0: 0 \leqslant e_{i j}(k) \leqslant C \tau^{k}, \quad C>0,
$$

где $\tau$ было определено в лемме 2 и $1 \leqslant i, j \leqslant|S|$.

Лемма 3. Если выполнено предположение 1, то максимальное по модулю собственное значение $\varrho^{*}$ матрицы $Q$ равно 1.

Д о к а з а т е л ь с т в о. Обозначим через $\varrho^{*}(t)$ максимальное по модулю собственное значение матрицы $Q-E(t)$. Будем доказывать от противного.

Пусть $\varrho^{*}>1$, тогда в силу (17) существует $T>0$ такое, что $\forall t>T$ : $\varrho^{*}(t)>\varrho^{\prime}>1$. Тогда из (16) и теоремы Перрона-Фробениуса следует, что существуют $T_{0}>T, s \in S$, для которых $\vec{r}\left(s, T_{0}\right)>1$, что невозможно.

Пусть $\varrho^{*}<1$, тогда в силу (17) существует $T>0$ такое, что $\forall t>T: \varrho^{*}(t)<\varrho^{\prime}<$ 1. Но тогда из (16) и теоремы Перрона-Фробениуса следует, что

$$
\forall s \in S: \lim _{t \rightarrow \infty} \vec{r}(s, t)=0,
$$

а это противоречит тому, что в надкритическом случае $q^{[1]}, \ldots, q^{[|S|]}<1$ (см. $[5$, c. 41]).

\section{Следовательно, $\varrho^{*}=1$.}

Из оценки (17), леммы 3 и критерия сходимости произведений матриц (см. [6, c. 416] или [3, с. 187]) следует сушествование предела для (16) при $t \rightarrow \infty$

$$
r^{[i]}(s)=\lim _{t \rightarrow \infty} r^{[i]}(s, t), \quad i=1, \ldots,|S| .
$$

В случае $|\alpha| \geqslant 2$ рекуррентные соотношения на $\vec{r}(\alpha, t), t>0$ принимают вид

$$
\vec{r}(\alpha, t)=(Q-E(t-1)) \vec{r}(\alpha, t-1)+\vec{\delta}(\alpha, t-1),
$$


где матрицы $Q$ и $E(t-1)$ были определены выше и $\vec{\delta}(\alpha, T), T \geqslant 0$, есть вектор с компонентами $\delta^{[k]}(\alpha, T), k=1, \ldots,|S|$, которые определяются следуюшим образом (см. pис. 1): $\delta^{[k]}(\alpha, 0)=\sum_{\beta \in S^{*}} \mathbf{P}\left\{s_{k} \rightarrow \alpha \beta\right\}$,

$$
\begin{aligned}
\forall T>0: \delta^{[k]}(\alpha, T)= & \sum_{m=2}^{K} \sum_{i_{1}, \ldots, i_{m}=1}^{|S|} \mathbf{P}\left\{s_{k} \rightarrow s_{i_{1}} \ldots s_{i_{m}}\right\} \\
& \times \sum_{n=2}^{m} \sum_{\left(\lambda_{1} \ldots \lambda_{n}\right) \in A(n, \alpha)} \prod_{j=1}^{n} \mathbf{P}\left\{\gamma^{\left[i_{j}\right]}(T)=\lambda_{j}\right\},
\end{aligned}
$$

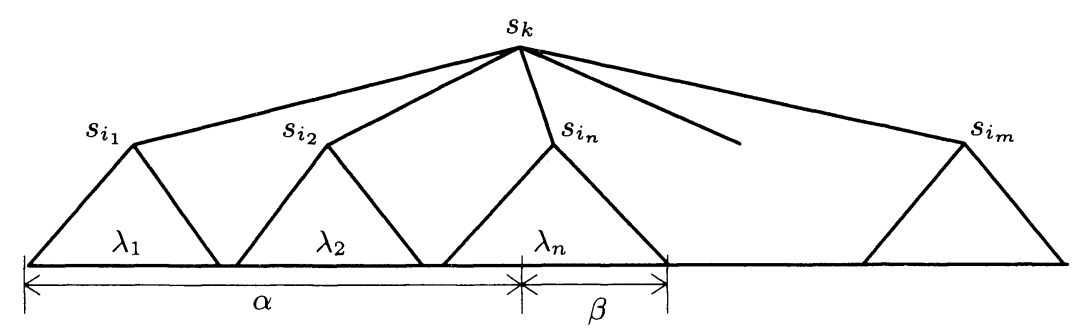

Рис. 1. Пояснение к определению $\delta^{[k]}(\alpha, T)$.

где

$$
\begin{aligned}
A(n, \alpha)=\left\{\left(\lambda_{1}, \ldots, \lambda_{n}\right): \lambda_{i} \in S^{*}, i=1, \ldots, n,\right. & \\
& \left.\exists \beta \in S^{*}: \lambda_{1} \ldots \lambda_{n}=\alpha \beta, 0<\sum_{l=1}^{n-1}\left|\lambda_{l}\right|<|\alpha| \leqslant \sum_{l=1}^{n}\left|\lambda_{l}\right|\right\} .
\end{aligned}
$$

Итерируя (19), с учетом $\vec{r}(\alpha, 0)=\mathbf{0}$ получим: $\vec{r}(\alpha, 1)=\vec{\delta}(\alpha, 0)$,

$$
\forall t \geqslant 2: \vec{r}(\alpha, t)=\sum_{i=1}^{t-1} \prod_{j=i}^{t-1}(Q-E(j)) \vec{\delta}(\alpha, i-1)+\vec{\delta}(\alpha, t-1) .
$$

Из определения $\delta^{[k]}(\alpha, t)$ и леммы 2 следует, что существуют $C>0$ и $\tau<1$ такие, что при $t>0$ верна оценка

$$
\delta^{[k]}(\alpha, t) \leqslant c^{\prime} \max _{1 \leqslant a \leqslant|S|}\left\{\mathbf{P}\left\{0<n^{[a]}(t)<|\alpha|\right\}\right\}<C \tau^{t}, \quad k=1, \ldots,|S|, \quad c^{\prime}>0 .
$$

Тогда, в силу оценки (17) и критерия сходимости произведения матриц, ряд в правой части (20) сходится, а значит, предел $\vec{r}(\alpha, t)$ при $t \rightarrow \infty$ сушествует. Для дальнейшего доказательства теоремы 1 нам понадобится следующее определение.

О п р е д е л е н и е 4 . В процессе $Z_{t}^{[\gamma]}$ символ $x_{j}^{[\gamma]}(t+T), j>0, t \geqslant 0$, является потомком символа $x_{i}^{[\gamma]}(t), i>0$, в момент времени $t+T$ (обозначается $x_{i}^{[\gamma]}(t) \Rightarrow$ $\left.x_{j}^{[\gamma]}(t+T)\right)$, если

a) при $T=1$ символ $x_{i}^{[\gamma]}(t)$ перешел в слово $x_{m}^{[\gamma]}(t+1) \ldots x_{n}^{[\gamma]}(t+1)$ и $m \leqslant j \leqslant n$, где $m, n>0$;

б) при $T>1$ сушествуют $i_{1}, \ldots, i_{T-1}>0$ такие, что $x_{i}^{[\gamma]}(t) \Rightarrow x_{i_{1}}^{[\gamma]}(t+1) \Rightarrow \cdots \Rightarrow$ $x_{i_{T-1}}^{[\gamma]}(t+T-1) \Rightarrow x_{j}^{[\gamma]}(t+T)$.

Для процесса $Z_{t}^{[k]}, k=1, \ldots,|S|$ понятие $x_{i}^{[k]}(t) \Rightarrow x_{j}^{[k]}(t+T)$ определяется аналогично для $t \geqslant 0, i, j, T>0$.

Введем $\beta_{i}^{[\gamma]}(t)=x_{a(i, t)}^{[\gamma]}(t) \ldots x_{b(i, t)}^{[\gamma]}(t), i>0$, где $a(i, t)=\min _{j}\left\{j: x_{i}^{[\gamma]}(0) \Rightarrow x_{j}^{[\gamma]}(t)\right\}$, $b(i, t)=\max _{j}\left\{j: x_{i}^{[\gamma]}(0) \Rightarrow x_{j}^{[\gamma]}(t)\right\}$. Если не сушествует $j>0$ такого, что $x_{i}^{[\gamma]}(0) \Rightarrow$ 
$x_{j}^{[\gamma]}(t)$, то $\beta_{i}^{[\gamma]}(t)=e$. Таким образом, $\beta_{i}^{[\gamma]}(t)$ состоит из всех потомков символа $x_{i}^{[\gamma]}(0)$ в момент времени $t$. Введем также векторы размерности $|S|: \vec{g}_{n}^{[\gamma]}=(0, \ldots, 0,1,0, \ldots, 0)$, $n>0$, где 1 стоит на $i$-м месте, если $x_{n}^{[\gamma]}(0)=s_{i} \in S, 1 \leqslant i \leqslant|S|$, и величину $q_{n}^{[\gamma]}(t)$, $n, t>0$, равную $q^{[i]}(t)$, если $x_{n}^{[\gamma]}(0)=s_{i}$. Тогда для $\alpha \in S^{+}$и $t>0$ :

$$
p^{[\gamma]}(\alpha, t)=\left(\vec{g}_{1}^{[\gamma]} \cdot \vec{r}(\alpha, t)\right)+\sum_{n=2}^{\infty} q_{1}^{[\gamma]}(t) \cdots q_{n-1}^{[\gamma]}(t)\left(\vec{g}_{n}^{[\gamma]} \cdot \vec{r}(\alpha, t)\right)+\phi^{[\gamma]}(\alpha, t),
$$

где $\phi^{[\gamma]}(\alpha, t)=\sum_{n=2}^{\infty} \sum_{\left(\lambda_{1}, \ldots, \lambda_{n}\right) \in A(n, \alpha)} \prod_{j=1}^{n} \mathbf{P}\left\{\beta_{j}^{[\gamma]}(t)=\lambda_{j}\right\}$. Из леммы 2 следует, что сушествуют $C>0$ и $\tau<1$ такие, что $\forall t>0$ :

$$
\begin{aligned}
\phi^{[\gamma]}(\alpha, t) & \leqslant \mathbf{P}\left\{0<\left|\beta_{1}^{[\gamma]}(t)\right|<|\alpha|\right\}+\sum_{m=1}^{\infty} q_{1}^{[\gamma]}(t) \cdots q_{m}^{[\gamma]}(t) \mathbf{P}\left\{0<\left|\beta_{m+1}^{[\gamma]}(t)\right|<|\alpha|\right\} \\
& \leqslant\left(1+\sum_{m=1}^{\infty} q_{\max }^{m}\right) \max _{1 \leqslant a \leqslant|S|}\left\{\mathbf{P}\left\{0<n^{[a]}(t)<|\alpha|\right\}\right\}<C \tau^{t},
\end{aligned}
$$

где $q_{\max }=\max _{i=1, \ldots,|S|} q^{[i]}<1$, так как в положительно регулярном надкритическом случае $q^{[i]}<1, i=1, \ldots,|S|$ (см. [5, с. 41]). Следовательно ряд $\sum_{m=1}^{\infty} q_{\max }^{m}$ сходится как геометрическая прогрессия.

Ряд в правой части (21) оценивается сверху рядом $\sum_{m=1}^{\infty} q_{\max }^{m}$ и, следовательно, сходится равномерно по $t$, а значит, в силу существования пределов $\lim _{t \rightarrow \infty} q_{n}^{[\gamma]}(t)=$ $q_{n}^{[\gamma]}<1, n=1,2, \ldots$, и $\lim _{t \rightarrow \infty} \vec{r}(\alpha, t)=\vec{r}(\alpha)$ можно перейти к почленному пределу при $t \rightarrow \infty$ в ряде в правой части (21), учитывая (22), получим

$$
\pi^{[\gamma]}(\alpha)=\lim _{t \rightarrow \infty} p^{[\gamma]}(\alpha, t)=\left(\vec{g}_{1}^{[\gamma]} \cdot \vec{r}(\alpha)\right)+\sum_{n=2}^{\infty} q_{1}^{[\gamma]} \cdots q_{n-1}^{[\gamma]}\left(\vec{g}_{n}^{[\gamma]} \cdot \vec{r}(\alpha)\right) .
$$

Теорема 1 полностью доказана.

Доказательство теоремы 2. Для любого слова $\alpha \in S^{+}$и $t>0$ введем $e^{[k]}(t, \alpha)=\mathbf{E} n\left(\alpha, \gamma^{[k]}(t)\right), e^{[k]}(0, \alpha)=0, k=1, \ldots,|S|$. Наша цель - написать рекуррентные уравнения для $e^{[k]}(t, \alpha)$ и исследовать их асимптотическое решение при $t \rightarrow \infty$. Введем $\varepsilon^{[k]}(t, \alpha), k=1, \ldots,|S|, t \geqslant 0$, следуюшим образом: $\varepsilon^{[k]}(0, \alpha) \equiv 0$,

$$
\varepsilon^{[k]}(1, \alpha)=e^{[k]}(1, \alpha)=\sum_{\beta \in S^{+}} n(\alpha, \beta) \mathbf{P}\left\{s_{k} \rightarrow \beta\right\} .
$$

Для определения $\varepsilon^{[k]}(t, \alpha)$ при $t>1$ разобьем слово $\gamma^{[k]}(t)$ на непересекающиеся подслова $\lambda_{i}^{[k]}(t)$ :

$$
\lambda_{i}^{[k]}(t)=x_{a(i, k, t)}^{[k]}(t) \ldots x_{b(i, k, t)}^{[k]}(t), \quad i=1, \ldots, n^{[k]}(1)
$$

где

$$
a(i, k, t)=\min _{j}\left\{j: x_{i}^{[k]}(1) \Rightarrow x_{j}^{[k]}(t)\right\}, \quad b(i, k, t)=\max _{j}\left\{j: x_{i}^{[k]}(1) \Rightarrow x_{j}^{[k]}(t)\right\} .
$$

Если не существует $j>0$ такого, что $x_{i}^{[k]}(1) \Rightarrow x_{j}^{[k]}(t)$, то $\lambda_{i}^{[k]}(t)=e$. Таким образом, $\lambda_{i}^{[k]}(t), i=1, \ldots, n^{[k]}(1), t>1$, состоит из всех потомков символа $x_{i}^{[k]}(1)$ в момент времени $\dot{t}$.

Для любого слова $\alpha=y_{1} \ldots y_{|\alpha|},|\alpha|>0, y_{1}, \ldots, y_{|\alpha|} \in S$, обозначим через $\varepsilon^{[k]}(t, \alpha)$, $k=1, \ldots,|S|, t \geqslant 2$, математическое ожидание числа подслов $x_{j}^{[k]}(t) \ldots x_{j+|\alpha|-1}^{[k]}(t)=\alpha$, $1 \leqslant j \leqslant n^{[k]}(t)-|\alpha|+1$, слова $\gamma^{[k]}(t)$, содержащих в себе потомков не менее двух 
символов $x_{i}^{[k]}(1), i=1, \ldots, n^{[k]}(1)$, т.е. не являющихся подсловом ни одного $\lambda_{i}^{[k]}(t)$, $i=1, \ldots, n^{[k]}(1)$ :

$$
\begin{gathered}
\varepsilon^{[k]}(t, \alpha)=\sum_{j>0} \mathbf{P}\left\{n^{[k]}(t) \geqslant j+|\alpha|-1 ; x_{j}^{[k]}(t)=y_{1}, \ldots, x_{j+|\alpha|-1}^{[k]}(t)=y_{|\alpha|} ;\right. \\
\left.x_{j}^{[k]}(t) \ldots x_{j+|\alpha|-1}^{[k]}(t) \not \subset \lambda_{i}^{[k]}(t), i=1, \ldots, n^{[k]}(1)\right\},
\end{gathered}
$$

где $\beta_{1} \not \subset \beta_{2}$ означает, что слово $\beta_{1} \in S^{*}$ не является подсловом слова $\beta_{2} \in S^{*}$.

Величина $\varepsilon^{[k]}(t, \alpha), t>1$, оценивается сверху максимальным числом подслов длины $|\alpha|$ слова $\gamma^{[k]}(t)$, содержащих в себе потомков не менее двух символов $x_{i}^{[k]}(1)$, $i=1, \ldots, n^{[k]}(1)$. Так как из $(2)$ следует, что $n^{[k]}(1) \leqslant K$, то

$$
\forall \alpha \in S^{+}, \forall t>1: \varepsilon^{[k]}(t, \alpha) \leqslant(|\alpha|-1)(K-1), \quad \varepsilon^{[k]}(1, \alpha) \leqslant K,
$$

$k=1, \ldots,|S|$. Эта оценка нам понадобится ниже.

Напишем рекуррентные уравнения для $e^{[k]}(t, \alpha), k=1, \ldots,|S|, t>0$ :

$$
e^{[k]}(t, \alpha)=\sum_{\beta \in S^{+}} \mathbf{P}\left\{s_{k} \rightarrow \beta\right\} \sum_{j=1}^{|S|} n\left(s_{j}, \beta\right) e^{[j]}(t-1, \alpha)+\varepsilon^{[k]}(t, \alpha) .
$$

Матрица с компонентами $m_{i j}=\sum_{\beta \in S^{+}} \mathbf{P}\left\{s_{i} \rightarrow \beta\right\} n\left(s_{j}, \beta\right), i, j=1, \ldots,|S|$, есть в точности матрица средних $M$ ветвящегося процесса $\vec{N}_{t}$. Тогда, обозначив через $\vec{e}(t, \alpha)$ и $\vec{\varepsilon}(t, \alpha)$ векторы с компонентами $e^{[i]}(t, \alpha)$ и $\varepsilon^{[i]}(t, \alpha), i=1, \ldots,|S|$, соответственно, мы можем переписать (24) в векторной форме:

$$
\vec{e}(t, \alpha)=M \cdot \vec{e}(t-1, \alpha)+\vec{\varepsilon}(t, \alpha) .
$$

Итерируя это уравнение, с учетом $\vec{e}(0, \alpha)=\mathbf{0}$ получим, что

$$
\forall t>0: \vec{e}(t, \alpha)=\sum_{i=0}^{t-1} M^{i} \vec{\varepsilon}(t-i, \alpha) .
$$

Исследуем поведение $\vec{e}(t, \alpha)$ при $t \rightarrow \infty$. Из теоремы Перрона-Фробениуса (см. [5, c. 38] или [3, с. 185]) следует, что $M^{t}=\varrho^{t} P+R^{t}$, где $P$ имеет строго положительные компоненты $u_{i} v_{j}, \vec{u}=\left(u_{1}, \ldots, u_{|S|}\right)$ есть правый, а $\vec{v}=\left(v_{1}, \ldots, v_{|S|}\right)$ - левый собственные векторы матрицы $M$, соответствующие ее максимальному собственному значению $\varrho>1$, с нормировкой: $(\vec{u} \cdot \vec{v})=1,(\vec{u} \cdot \mathbf{1})=1$, а матрица $R$ такова, что модули компонент матрицы $R^{t}$ не превосходят $C \varrho_{0}^{t}, C>0, \varrho_{0}<\varrho$. Подставим это в (25):

$$
\frac{\vec{e}(t, \alpha)}{\varrho^{t}}=\frac{\sum_{i=0}^{t-1} \varrho^{i} P \vec{\varepsilon}(t-i, \alpha)}{\varrho^{t}}+\frac{\sum_{i=0}^{t-1} R^{i} \vec{\varepsilon}(t-i, \alpha)}{\varrho^{t}} .
$$

Так как модули компонент матрицы $R^{i}$ при $i \geqslant 0$ не превосходят $C \varrho_{0}^{i}, C>0, \varrho_{0}<\varrho$, то, учитывая (23), получаем, что второе слагаемое в (26) стремится к нулю при $t \rightarrow \infty$. Рассмотрим первое слагаемое. Так как $\varrho>1$, то, учитывая (23), получим

$$
P \sum_{j=1}^{t} \frac{\vec{\varepsilon}(j, \alpha)}{\varrho^{j}} \underset{t \rightarrow \infty}{\longrightarrow}\left(\begin{array}{ccc}
u_{1} v_{1} & \ldots & u_{1} v_{|S|} \\
\vdots & & \vdots \\
u_{|S|} v_{1} & \ldots & u_{2} v_{|S|}
\end{array}\right)\left(\begin{array}{c}
L^{[1]}(\alpha) \\
\vdots \\
L^{[|S|]}(\alpha)
\end{array}\right)
$$

где $L^{[1]}(\alpha), \ldots, L^{[|S|]}(\alpha)>0-$ константы, зависящие только от слова $\alpha$. Обозначив $d(\alpha)=\sum_{i=1}^{[S \mid} v_{i} L^{[i]}(\alpha)>0$, из $(26)$ и $(27)$ получаем, что

$$
\lim _{t \rightarrow \infty} e^{[k]}(t, \alpha) \varrho^{-t}=u_{k} d(\alpha), \quad k=1, \ldots,|S| .
$$


С другой стороны, так как $E\left(\vec{N}_{t}^{[k]}\right)=\mathbf{e}_{k} M^{t}$, то для $n^{[k]}(t)=\sum_{i=1}^{|S|} N_{t}^{[k]}\left(s_{i}\right)$ из теоремы Перрона-Фробениуса получаем

$$
\lim _{t \rightarrow \infty} \frac{\mathbf{E} n^{[k]}(t)}{\varrho^{t}}=u_{k} \sum_{i=1}^{|S|} v_{i}, \quad k=1, \ldots,|S| .
$$

Тогда из определения $\mu^{[k]}(\alpha ; t), k=1, \ldots,|S|$, получаем

$$
\mu(\alpha)=\lim _{t \rightarrow \infty} \mu^{[k]}(\alpha ; t)=\frac{u_{k} d(\alpha)}{u_{k}\left(v_{1}+\cdots+v_{|S|}\right)}=\frac{d(\alpha)}{v_{1}+\cdots+v_{|S|}}, \quad k=1, \ldots,|S| .
$$

Таким образом, теорема 2 полностью доказана.

\section{СПИСОК ЛИТЕРАТУРЫ}

1. Малыщев В. А. Случайные грамматики. - Успехи матем. наук, 1998, т. 53, № 2 , c. $107-134$.

2. Севастьянов Б. А. Ветвящиеся процессы. М.: Наука, 1971.

3. Athreya K. B., Ney P.E. Branching Processes. Berlin-Heidelberg: Springer-Verlag, $1972,287 \mathrm{p}$.

4. Athreya K. B., Vidyashankar A. N. Large deviation results for branching processes. II: The multitype case. - Ann. Appl. Probab., 1995, v. 5, № 2, p. 566-576.

5. Harris T.E. The Theory of Branching Processes. Berlin-Heidelberg: Springer-Verlag, $1963,230 \mathrm{p}$.

6. Joffe A., Spitzer F. On multitype branching process with $\varrho<1$. - J. Math. Anal. Appl., 1967, v. 19, № 3, p. 409-430.

7. Karpelevich F.I., Malyshev V.A., Petrov A.I., Pirogov S.A., Rybko A. N. Context Free Evolution of Words. - Rapport de Recherche № 4413. Rocquencourt: INRIA, 2002.

Поступила в редакцию

19.II.2002

(c) 2002 r.

ПИТЕРБАРГ В. И. ${ }^{*}$ К КОЗЛОВ А. М.*

\section{О БОЛЬШИХ СКАЧКАХ СЛУЧАЙНОГО БЛУЖДАНИЯ С УСЛОВИЕМ КРАМЕРА ${ }^{1)}$}

В работе получена асимптотика вероятностей больших уклонений для приращений случайного блуждания с условием Крамера.

Ключевые слова и фразы: случайное блуждание, большие уклонения, условие Крамера.

* Московский государственный университет им. М.В. Ломоносова, механикоматематический факультет, Ленинские горы, 119992 Москва, Россия; е-mail: piter@mech.math.msu.su

1) Работа выполнена при частичной поддержке РФФИ (гранты №01-01-00649, 00-01-00247, 01-01-00644). 